\title{
Microarray analysis reveals a potential role of LncRNAs expression in cardiac cell proliferation
}

\author{
Jue Wang ${ }^{1 \dagger}$, Zhimin Geng ${ }^{2,3+}$, Jiakan Weng ${ }^{1}$, Longjie Shen ${ }^{4}$, Ming Li ${ }^{5}$, Xueli Cai ${ }^{6}$, Chengchao Sun ${ }^{1 *}$
} and Maoping $\mathrm{Chu}^{2^{*}}$ (D)

\begin{abstract}
Background: Long non-coding RNAs (LncRNAs) have been identified to play important roles in epigenetic processes that underpin organogenesis. However, the role of LncRNAs in the regulation of transition from fetal to adult life of human heart has not been evaluated.

Methods: Immunofiuorescent staining was used to determine the extent of cardiac cell proliferation. Human LncRNA microarrays were applied to define gene expression signatures of the fetal (13-17 weeks of gestation, $n=4)$ and adult hearts (30-40 years old, $n=4$ ). Pathway analysis was performed to predict the function of differentially expressed mRNAs (DEM). DEM related to cell proliferation were selected to construct a IncRNA-mRNA co-expression network. Eight IncRNAs were confirmed by quantificational real-time polymerase chain reaction $(n=6)$.

Results: Cardiac cell proliferation was significant in the fetal heart. Two thousand six hundred six IncRNAs and 3079 mRNAs were found to be differentially expressed. Cell cycle was the most enriched pathway in down-regulated genes in the adult heart. Eight IncRNAs (RP11-119 F7.5, AX747860, HBBP1, LINC00304, TPTE2P6, AC034193.5, XLOC_006934 and AL833346) were predicted to play a central role in cardiac cell proliferation.

Conclusions: We discovered a profile of IncRNAs differentially expressed between the human fetal and adult heart. Several meaningful IncRNAs involved in cardiac cell proliferation were disclosed.
\end{abstract}

Keywords: Long non-coding RNA, Microarray, Human heart, Cardiac cell proliferation

\section{Background}

The heart is the first-formed organ during human embryogenesis. Approximately 10 weeks after fertilization, four important processes, namely, looping, trabeculation, septation and myocardial compaction, have been completed in the human fetal heart. Through the remaining pregnancy period, the myocardium undergoes specialization into specific cardiac tissues. Previous studies have elucidated that the architecture of

\footnotetext{
* Correspondence: suncc6@163.com; chmping@hotmail.com

${ }^{\dagger}$ Equal contributors

'Department of Cardiac Surgery, the First Affiliated Hospital of Wenzhou Medical University, Nanbaixiang, Shangcaicun, Wenzhou 325000, Zhejiang Province, People's Republic of China

${ }^{2}$ Children's Heart Center, the Second Affiliated Hospital \& Yuying Children's Hospital, Institute of Cardiovascular Development and Translational Medicine, Wenzhou Medical University, No. 109, Xueyuan Road, Wenzhou 325000, Zhejiang Province, People's Republic of China

Full list of author information is available at the end of the article
}

human myocardium changes from isotropic to anisotropic during development [1]. During the fetal period, the major source of energy is glucose for the heart, which is different from a full utilization of fatty acid oxidation in the adult period [2]. Moreover, cardiomyocyte proliferation is evident during fetal life, but the proliferation capacity decreases in the adult heart [3]. During the transition from fetal to adult life of the heart, sophisticated regulatory networks are required to adapt to diverse physiological and pathophysiological responses. Recently, it has become evident that LncRNAs may in fact play major roles in most aspects of gene regulation, especially in the epigenetic processes that underpin organogenesis [4].

LncRNAs are long non-coding RNAs, which can function as either primary or spliced transcripts, and they are independent of the currently known classes of small 
RNAs (micro RNAs, piwi-interacting RNAs, and others) and exclude classic housekeeping families of RNAs (such as tRNAs and rRNAs) [5]. In recent years, accumulating evidence has indicated that lncRNAs are involved in a series of vital biological processes, such as serving as precursors for smaller RNAs and controlling the cell cycle [6], cell apoptosis [7], brain and testis development as well $[8,9]$. It has been reported that IncRNAs can impact gene expression at multiple levels (e.g., transcriptional and post-transcriptional control, epigenetic regulation). Moreover, lncRNAs have been found to be able to regulate the expressions of proximal and distal protein-coding genes through cis- and trans-acting mechanisms [10]. Until now, many lncRNAs have been identified in humans, which prompt the building of human IncRNA database providing expression profiles of lncRNAs [11].

Currently, several lncRNAs have been shown to exert critical roles in cardiac development and regeneration, such as lncRNA-Braveheart [12], IncRNA-Fendrr [13] and IncRNA-ST8SIA3 [14]. However, most of the literature on cardiac profiling is based on animal models, such as mice. As there is a great difference between human heart and animal heart, and lncRNA sequences generally exhibit low conservation across species [15], it is requisite to determine the IncRNA profile in human fetal and adult heart and find key lncRNAs that regulate the transition from fetal to adult life of the heart. To this end, we have used high-throughput microarray lncRNA screening to investigate differentially expressed lncRNAs and mRNAs between human fetal hearts and adult hearts. As a result, pathways analysis revealed that the down-regulated genes in the adult group were mainly related to cell proliferation. Particular lncRNAs were predicted to play important roles in cardiac cell proliferation.

\section{Methods}

\section{Tissue collection}

The fetal cardiac tissues used in this study were collected from patients undergoing prostaglandin induction of labor for termination of pregnancy due to unwanted pregnancy. Six fetal hearts were obtained, and the gestational ages were between 13 and 17 weeks. Adult cardiac tissues were collected from six unused organ donor hearts aged between 30 and 40 years old. The heart was carefully separated from the rest of the thoracic content with sterilized surgical instruments. After dissection, the heart was investigated and confirmed to have normal cardiac anatomy and then washed with PBS buffer (Ambion, USA). Myocardial samples were taken from the left ventricle and immediately stored in liquid nitrogen.

\section{Immunofiuorescent staining}

The fetal and adult heart tissues were fixed in 4\% paraformaldehyde. Sections of myocardial tissues were mounted onto glass slides. For immunofiuorescent staining, the sections were permeabilized in $0.3 \%$ Triton $\mathrm{X}$ 100 in PBS solution for $5 \mathrm{~min}$, followed by blocking in $5 \%$ goat serum plus $5 \%$ donkey serum for $1 \mathrm{~h}$. Then the sections were stained with the primary antibodies overnight at $4{ }^{\circ} \mathrm{C}$. The primary antibodies were Mouse anticardiac Troponin T antibody (cTnT, Abcam) and Rabbit anti-Ki-67 antibody (Abcam), which diluted in blocking solution (1:200). The appropriate secondary antibodies conjugated to DyLight-594 and Fitc-488 (Abcam) were added to the tissues for $1 \mathrm{~h}$ at room temperature and the tissues were washed with PBST (PBS buffer with $0.05 \%$ Tween 20) for $10 \mathrm{~min}$ and PBS for $5 \mathrm{~min}$. The cell nuclei were counter-stained with 4, 6-diamidino-2-phenylindole (DAPI, Beyotime) for $2 \mathrm{~min}$ and then covered with $70 \%(\mathrm{v} / \mathrm{v})$ glycerol. After mounted with glass cover slips, the immunofiuorescent staining was captured under a laser confocal fluorescence microscope. (Nikon, A1).

\section{RNA extraction}

RNA was extracted from frozen myocardial tissue using Trizol reagent (Invitrogen Life Technologies, Carlsbad, CA, USA). The quantification and quality of the extracted RNA were measured using a Nano Drop ND1000 spectrophotometer. The high purity of the isolated RNA, as indicated by $A 260 / 280 \geq 1.90$, was confirmed before microarray and quantificational real-time polymerase chain reaction (qRT-PCR) experiments.

\section{Microarray analysis}

RNA samples were analyzed through the microarray. An Arraystar Human LncRNA Microarray v $3.0(8 \times 60 \mathrm{~K}$, Arraystar) was used, which can detect 30,586 lncRNAs and 26,109 protein-coding transcripts. Briefly, rRNA was removed from total RNA, and then, mRNA was obtained (mRNA-ONLY ${ }^{\mathrm{mi}}$ Eukaryotic mRNA Isolation Kit, Epicentre). The random priming method was utilized to amplify each sample, and mRNA was transcribed into fluorescent cRNAs without 3'bias. Labeled cRNAs were hybridized to the Human LncRNA Microarray. An Agilent Microarray Scanner (Agilent p/n G2565BA) was used to scan the microarray after the slides were washed. The acquired array images were analyzed using Agilent Feature Extraction software (version 11.0.1.1). After performing quantile normalization, IncRNAs and mRNAs for which at least four of the eight samples had present or marginal flags ("All Targets Value") were chosen for further data analysis using the GeneSpring GX v11.5.1 software package (Agilent Technologies). 


\section{Pathway analysis}

DEM were uploaded into the Kyoto Encyclopedia of Genes and Genomes database (KEGG, http://www.genome.ad.jp/kegg/). Pathway analysis was applied to analyze the potential pathways that DEM involved.

\section{Co-expression network construction}

To predict the key lncRNAs that function in the proliferation of cardiac cells, IncRNA-mRNA co-expression network was constructed on the basis of the correlation between the differentially expressed lncRNAs and mRNAs that related to cell proliferation. Pearson's correlation coefficient was calculated to measure the gene co-expression, and the absolute value $\geq 0.99975$ and $P$-value $<0.001$ were considered to be strong correlated between lncRNAs and mRNAs. In the network, a node represents a gene and two correlated genes are connected with an edge, with solid line representing positive correlation and dotted line representing negative correlation. Square nodes represent lncRNAs, while circular nodes represent mRNAs. Red nodes are symbols of down-regulated genes and blue ones are upregulated genes in the adult heart. Degree is used to describe the number of genes related to the key lncRNAs. The larger the node is, the higher the degree would be.

\section{qRT-PCR validation assay}

The SYBR green method was used to perform qRT - PCR in an Applied Biosystems 7300 Sequence Detection System (ABI 7300 SDS; Foster City, CA, USA). The reaction conditions of PCR included a denaturation step of $10 \mathrm{~min}$ at $95{ }^{\circ} \mathrm{C}$ and 40 cycles of $15 \mathrm{~s}$ at $95^{\circ} \mathrm{C}$ and $1 \mathrm{~min}$ at $60{ }^{\circ} \mathrm{C}$. The gene expression levels were normalized to the housekeeping gene GAPDH, and all samples were measured in triplicate. The relative expression levels of the genes were calculated using the $2^{-\Delta \Delta \mathrm{Ct}}$ method.

\section{Statistical analysis}

All of the data are shown as the mean \pm standard deviation. The statistical significance of differentially expressed lncRNAs between the fetal heart and the adult heart, were analyzed using Student's t-test in SPSS 16.0 (SPSS, Chicago, IL, USA). Fisher's exact test was used to examine the significance of the pathway correlated to the conditions, $P<0.05$ and FDR $<0.05$ were considered statistically significant. FDR was used to correct the $P$ values.

\section{Results}

\section{Cardiac cell proliferation in fetal and adult hearts}

We firstly used two antibodies (Ki-67 and cardiac Troponin $\mathrm{T}(\mathrm{c} \operatorname{Tn} \mathrm{T})$ ) to stain myocardium sections of both fetal and adult hearts to determine the extent of cardiac cell proliferation. Ki-67 has been reported to be detected in proliferating cells at all active phases of cell cycle while cTnT is a cardiomyocyte marker that expresses in both cycling and non-cycling cells. Here, Ki-67 stained cells were observed in fetal heart (Fig. 1a), not in adult heart (Fig. 1b), proving that the cardiac cell proliferation only occurred in fetal heart. Ki-67 positive cardiomyocytes and all Ki-67 positive cells were quantified. Percentage of Ki-67 positive cardiomyocytes in fetal myocardium is $67.73 \%$ while adult myocardium is zero (Fig. 1c).

\section{Differentially expressed LncRNAs and mRNAs}

LncRNA expression profiling data showed that 18,137 lncRNAs and 20,363 protein-coding RNAs were expressed using microarray analysis. Thus, we evaluated the absolute ratio of normalized intensities in paired samples (adult-to-fetal ratio, A/F). Two thousand six hundred six lncRNAs were found to be differentially expressed (fold change, $\mathrm{FC} \geq 2$ ) between two groups. One thousand three hundred forty five lncRNAs were up-regulated while 1261 were down-regulated in the
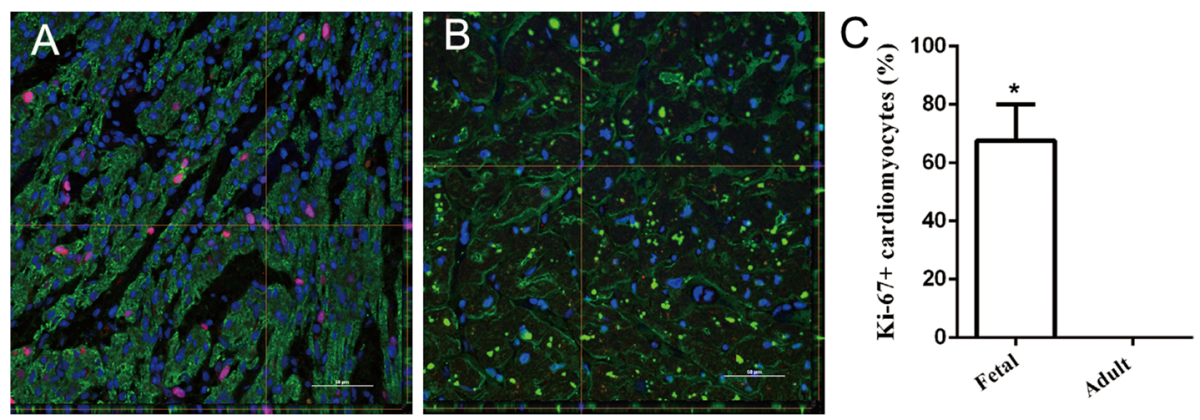

Fig. 1 Extent of cardiomyocyte proliferation in fetal and adult hearts. Immunofluorescent staining shows the proliferating Ki-67+ cardiac cell in fetal (a) and adult heart (b) respectively. Scale bar: $50 \mu \mathrm{m}$. The yellow lines indicate the position within the image of projections given in the lower and right. Percentage of Ki-67 positive cardiomyocyte in all positive cells was shown respectively (c). Data are expressed as the mean \pm standard deviation (SD) of three independent experiments $\left({ }^{*} P<0.01\right)$ 
adult group, compared with those in the fetal group (Additional file 1). A total of 357 lncRNAs were found to be significantly differentially expressed with a FC of five, among which 285 lncRNAs were down-regulated and 72 lncRNAs were up-regulated in the adult group, compared with the fetal group (Table 1). The top ten down-regulated and top ten up-regulated lncRNAs were listed in Table 2. Hierarchical clustering analysis has shown significantly up-regulated and down-regulated lncRNAs with $\mathrm{FC} \geq 5$ (Fig. 2a). A scatterplot was a visualization used to assess the variation between the chips (Fig. 2b). Additionally, 1446 mRNAs were downregulated, and 1633 were up-regulated in the adult group compared with the fetal group (Additional file 2).

\section{Pathway enrichment analysis}

Pathway enrichment analysis indicated that downregulated genes in the adult group were involved in 19 pathways, while the up-regulated genes were involved in 10 pathways. We have used - $\lg P$-value to describe significance level of the pathway enrichment (Fig. 3). The enriched pathways including "cell cycle pathway", "DNA replication", "p53 signaling pathway" and "Hippo signaling pathway", in down-regulated genes in the adult group, may have an important effect on cell proliferation. Among these pathways, the cell cycle pathway was the most enriched pathway related to the down-regulated genes.

The pathways involved in junction formation in cardiomyocyte such as "adherents junction" and "gap junction were also enriched in the down-regulated genes. In addition, calcium signaling pathway, which relates to the development of the cardiac conduction system, was represented in the up-regulated genes. Pathways relating to the immune system were flagged up in up-regulated genes. These included allograft rejection and graft-versus-host disease. The differentially expressed genes involved in the above pathways will provide a foundation for future study on the underlying mechanism of the changes that occur during the second trimester of heart development.

\section{LncRNA-mRNA co-expression network}

Previously published studies have demonstrated that IncRNAs play a critical role in gene transcription [16]. Here, we constructed a lncRNA-mRNA co-expression network to investigate how the differential expression of the lncRNAs involved cardiac cell proliferation. DEM involved in the pathways associated with cell proliferation

Table 1 Number of differentially expressed LncRNAs

\begin{tabular}{lllll}
\hline LncRNAs & FC $2-5$ & FC $\geq 5$ & FC $\geq 10$ & Total \\
\hline Down & 894 & 285 & 82 & 1261 \\
Up & 1259 & 72 & 14 & 1345 \\
\hline
\end{tabular}

were selected to establish a lncRNA-mRNA co-expression network. 242 pairs of lncRNAs and mRNAs were identified to be coexpressed (Additional file 3), with that 220 pairs were positively correlated and 22 pairs negatively correlated. XLOC_009300 - MMP1, AX747860 - ANLN, and RP11-119 F7.5 - DEFA3 were the highest positive correlated lncRNA-mRNA pairs, and these lncRNAs may promote the expression of the related mRNA. RP11119 F7.5 - PDLIM5, RP11-71H9.1 - ISCU and RP5$902 P 8.10$ - RPIA were the highest negative, this suggests that these lncRNAs may inhibit the expression of the related mRNA. LncRNA RP11-119 F7.5, AX747860, HBBP1, LINC00304, TPTE2P6, AC034193.5, XLOC_006934 and $A L 833346$, which were shown to have the highest degree, may play core roles in the co-expression network (Fig. 4). These lncRNA-mRNA pairs may play the crucial roles in the co-expression network.

\section{Microarray validation by qRT-PCR}

The eight focused lncRNAs, which were identified to be closely related with cardiac cell proliferation through lncRNA-mRNA co-expression network construction, were selected to verify their expression level and the microarray results using qRT-PCR with samples $(n=6$ /group). The qRT-PCR analysis showed that the eight IncRNAs changed significantly between the fetal and adult groups (Fig. 5a), and the expression tendency was confirmed to be concordant with the microarray data (Fig. 5b).

\section{Discussion}

In the current study, high-throughput microarray LncRNAs screening was utilized to identify differentially expressed lncRNAs between human fetal and adult hearts. Pathways relating to cell proliferation were most enriched in the down- regulated genes and pathways relating to immune system were most enriched in the up- regulated genes in the adult heart. Particular lncRNAs were predicted to play important roles in cardiac cell proliferation by the establishment IncRNAmRNA co-expression network.

Cardiomyocytes proliferation is important during heart development as it is required for normal heart morphogenesis and increases the heart size [17]. Although proliferation level of mammalian cardiomyocyte is high during fetal period, it starts to decrease in postnatal stages [18]. It has been found that the heart loses its regenerative ability which exists in the fetal heart when the cardiomyocyte proliferation declines in the adult heart $[19,20]$. Presently, we have demonstrated that cardiac cell proliferation was significant in the second trimester and cannot be detected in the adult heart by immunofiuorescent staining. Pathway analysis was performed on DEM and it revealed that down-regulated genes in the adult heart were mainly featured by the 
Table 2 Top 10 up-regulated and down-regulated LncRNAs

\begin{tabular}{|c|c|c|c|c|c|}
\hline \multicolumn{3}{|c|}{ Down-regulated LncRNAs } & \multicolumn{3}{|c|}{ Up-regulated LncRNAs } \\
\hline Sequence name & Gene symbol & FC & Sequence name & Gene symbol & FC \\
\hline ENST00000568019 & PWRN1 & 141.7670014 & ENST00000581502 & RP11-76 K13.3 & 357.5387068 \\
\hline ENST00000449258 & $R P 11-406023.2$ & 99.982081 & NR_027242 & SSTR5-AS1 & 31.9642591 \\
\hline ENSTO0000581798 & CTD-3096 M3.2 & 99.3375837 & TCONS_00007953 & XLOC_003422 & 15.8564648 \\
\hline ENST00000425771 & GAS5 & 70.4895431 & uc001loz.3 & BC040735 & 12.592759 \\
\hline ENST00000382864 & EGFEMTP & 68.5222621 & ENST00000523269 & MIR143HG & 12.5208372 \\
\hline ENST00000573315 & LINC00514 & 66.1058412 & ENST00000514473 & AE000661.37 & 12.2791441 \\
\hline ENST00000483846 & EGFEMIP & 65.7167316 & ENST00000556777 & AE000661.37 & 12.1201584 \\
\hline ENST00000554841 & RP1-261D10.2 & 63.4768112 & TCONS_00018898 & XLOC_008763 & 11.9494669 \\
\hline ENST00000445310 & KCNQ5-IT1 & 54.1499847 & TCONS_00013650 & XLOC_006306 & 11.6920115 \\
\hline ENST00000463978 & RP11-71H9.1 & 53.5463368 & ENST00000512263 & RP11-161D15.1 & 11.3482059 \\
\hline TCONS_00015370 & XLOC_006934 & 52.5315592 & ENST00000425802 & RP11-91A18.4 & 10.44422 \\
\hline
\end{tabular}

annotation "cell cycle". This finding suggests proliferative phenotype of cardiac cell in the second trimester, which collaborates with the immunohistological observations. These results are almost consistent with findings in mouse [21]. It has been reported that Hippo-deficient embryos had overgrown hearts with elevated cardiomyocyte proliferation in developing mouse heart [22].
However, our results showed that Hippo pathway was enriched in the down-regulated genes, which was different from the discovery in the mouse heart. Cardiac specific knockout of LATS2 and WW45 in mice resulted in over-grown hearts with elevated cardiomyocyte proliferation [22], but these two genes were not regulated in our result. In addition, YAP1, which is one of the key

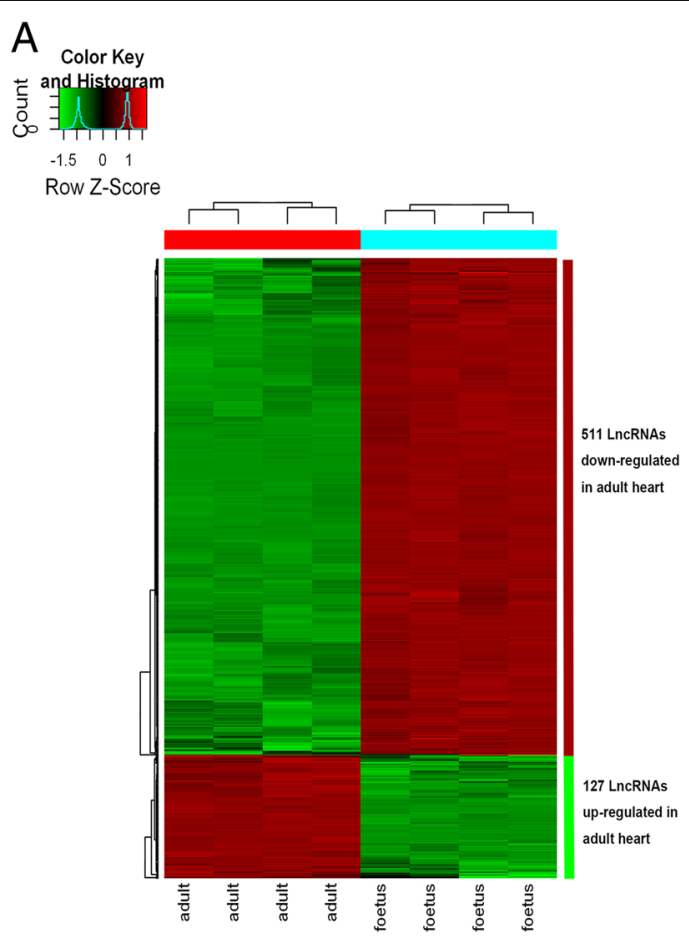

B

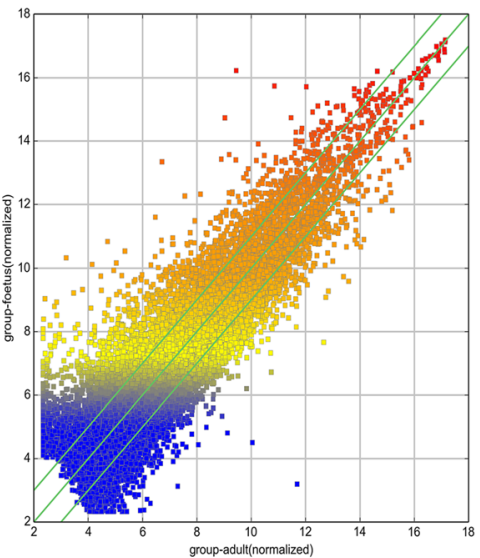

Fig. 2 Differential expression of IncRNAs between fetal and adult heart. Hierarchical clustering analysis shows differential expression of LncRNAs ( $F C \geq 5$ ) using a heat map (a). 'Red' indicates higher expression, whereas 'blue' indicates lower expression. The bar code represents the color scale of log 2 transformed values. The scatterplot (b) is a visualization used for assessing the variation between the chips. The $X$ and $Y$ axes in the scatterplot represent the normalized signal values of each group (log2 scaled). The green lines represent FC $(F C=2.0)$ lines 


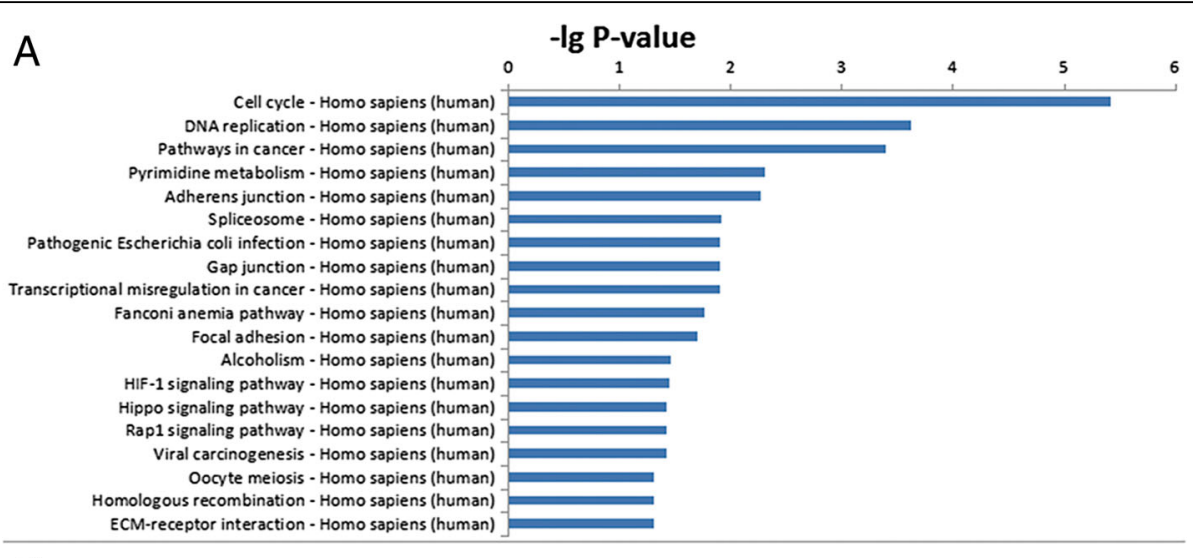

B

\section{-lg P-value}

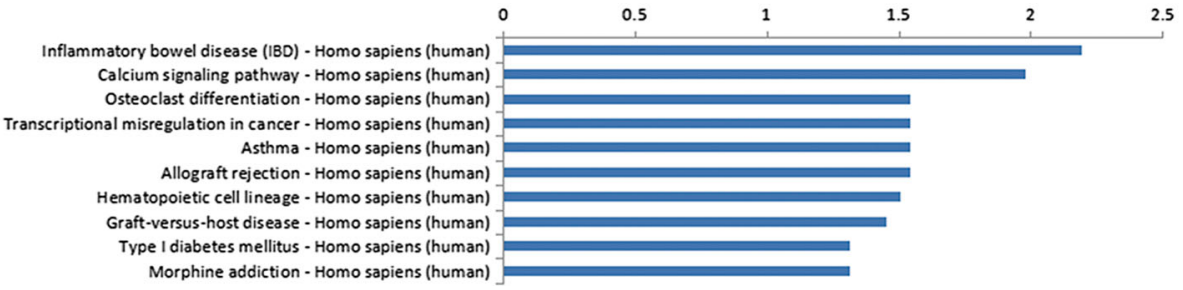

Fig. 3 Pathway analysis. The pathways that exhibited significant differences between fetal and adult hearts are listed. A shows the downregulated genes pathways and $B$ the up-regulated genes pathways in adult group. Higher -lg $P$-value indicates higher significance level

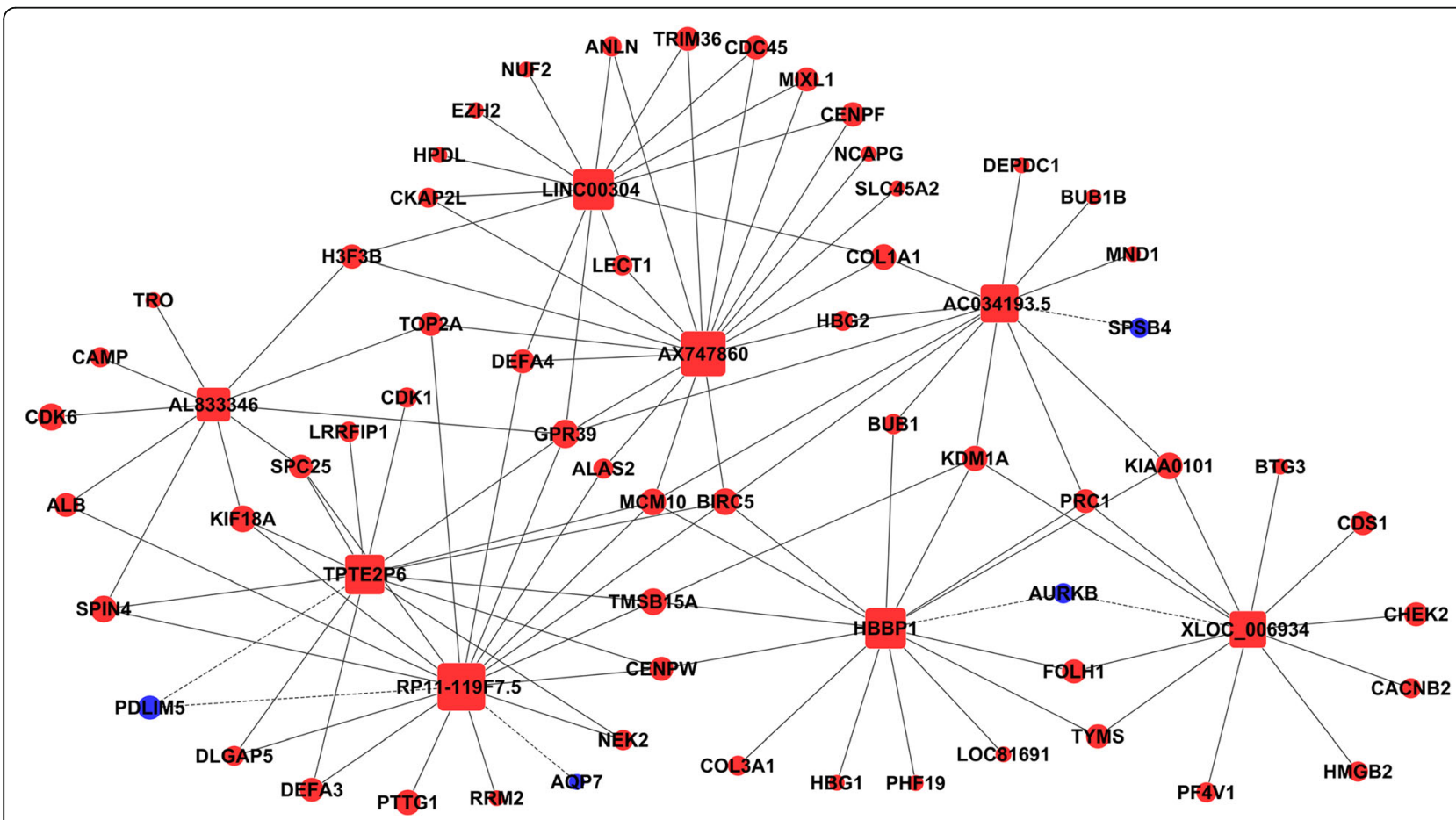

Fig. 4 Establishment of IncRNA-mRNA co-expression network. Nodes represent genes and an edge is used to connect two correlated genes. Solid lines represent positive correlation and dotted lines negative correlation. Blue nodes represent up-regulated genes and red nodes represent down-regulated genes in adult heart. Square nodes represent IncRNAs, while circular nodes represent mRNAs. The larger the node is, the higher the degree would be 

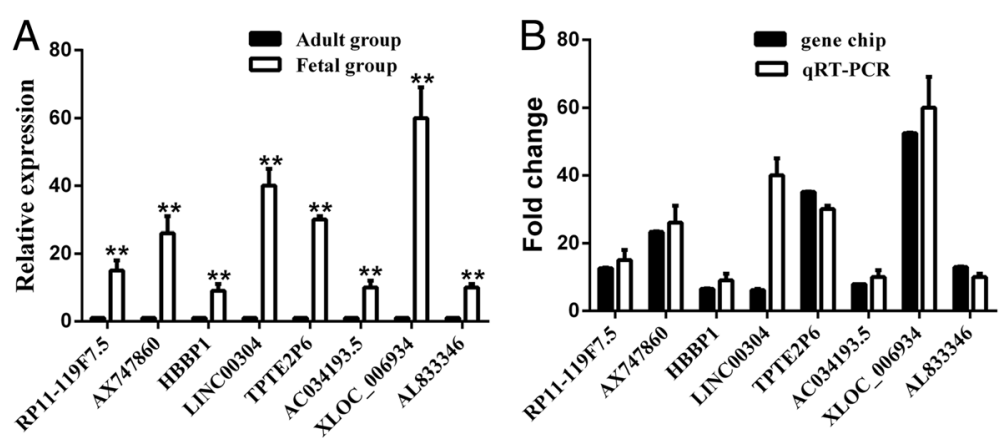

Fig. 5 qRT-PCR validation of differential expressions of LncRNAs.

a Eight LnCRNAs confirmed by qRT-PCR show to have significant changes between fetal and adult groups. Data are expressed as the mean \pm standard deviation (SD) of three independent experiments ( ${ }^{*} P<0.01$ ). $\mathbf{b}$ qRT-PCR patterns of seven LncRNAs except for LINC00304 are completely consistent with those of microarray data. Data of gene chip group are expressed as the mean \pm standard deviation (SD) $(n=4$, normalized data). The $Y$-axis represents FCs (A/F)

genes in Hippo pathway found in mouse heart [23, 24], was downregulated in the adult heart with FC of 5.59. This implied that YAP may also impede cardiomyocyte proliferation in the adult human heart.

What is more, allograft rejection pathway and graftversus-host disease pathway were involved significantly in the up-regulated genes in the adult heart. This result suggests that the activation of immune response in the second trimester, which was also found in the transition from 2- to 13-day-old mouse hearts [25].

Recently, increasing evidence has indicated that lncRNAs play significant roles in the proliferation of various cell types [26]. However, very few studies have been conducted on the potential functions of lncRNAs in cardiac cell proliferation. In the current study, 638 lncRNAs were found to be significantly differentially expressed $(\mathrm{FC} \geq 5.0)$ between the fetal and adult hearts. Most of the discovered lncRNAs were not functionally characterized, so lncRNA-mRNA coexpression network was constructed to predict the key lncRNAs that related to cell proliferation. LncRNA RP11119 F7.5, AX747860, HBBP1, LINC00304, TPTE2P6, $A C 034193.5, X L O C \_006934$ and AL833346, which were shown to have the highest degree, may play core roles in the co-expression network. The results imply that the regulation of these genes may affect cardiac cell proliferation through regulating the expression of their corresponding mRNAs.

Several significantly changed lncRNAs were predicted to be closely related to organ development and cardiac function. LncRNA ENST00000425771, a 242 nt lncRNA transcribed from a gene called growth arrest-specific 5 (GAS5), is a lncRNA located on chromosome 1. GAS5 can act as a riborepressor of the glucocorticoid receptor by binding to the DNA-binding domain of the glucocorticoid receptor [27], and glucocorticoids have been demonstrated to be critical for cardiac development [28]. Recently, increasing evidence has indicated that GAS5 plays a critical role in the proliferation of cancer cells [29, 30]. Therefore, we infer that the down-regulated IncRNA ENST00000425771 with a FC of 70.4895431 may function in the change in proliferation capacity of cardiomyocytes during cardiac development. LncRNA NR_038439 is a 1450 nt antisense transcript from the protein coding gene CACNA1G, and the FC of down-regulated lncRNA NR_038439 was 29.0475756. Previous findings show that $C A C$ $N A 1 G$, which is related to the T-type calcium currents, is mainly expressed in young and adult hearts $[31,32]$. This finding is consistent with our microarray data showing up-regulated expression of $C A C$ NA1G $(\mathrm{FC}=29)$ in the adult heart compared with the fetal heart. The expression of CACNA1G may be involved in hormone secretion [31].

\section{Conclusion}

In summary, we discovered for the first time a profile of IncRNAs differentially expressed between the human fetal and adult heart. Several meaningful lncRNAs involved in cardiac cell proliferation were disclosed. Although the mechanisms of the discovered lncRNAs in cardiac cell proliferation remain to be elucidated, we hope our novel discovery will lead to more studies that will determine its function.

In our future study, we will focus on the function of the above lncRNAs on several kinds of cardiac cells, such as cardiomyocytes, vascular endothelial cells, smooth muscle cells and cardiac fibroblasts, and hope to find the key factors that are able to promote cardiac cells proliferation. 


\section{Additional files}

Additional file 1: Differentially Expressed LncRNAs. (XLS $2.36 \mathrm{mb}$ )

Additional file 2: Differentially Expressed mRNAs. (XLS 4.45 mb)

Additional file 3: Coexpresses IncRNA-mRNA pairs. (PDF $126 \mathrm{~kb}$ )

\section{Abbreviations}

A/F: Adult-to-fetal ratio; DEM: Differentially expressed mRNAs; FC: Fold change; GAS5: Growth arrest-specific 5; LncRNA: Long non-coding RNA

\section{Acknowledgements}

This work was supported by National Natural Science Foundation of China (grant numbers: 81670255), Zhejiang Provincial Natural Science Foundation of China (grant numbers: LY14H020008), Wenzhou Science \& Technology Bureau (grant numbers: Y20130169), Science and Technology Department of Zhejiang Province (grant numbers: 2015C33163) and Zhejiang Provincial Natural Science Foundation of China (grant numbers: LY13H020005)

\section{Availability of data and materials}

The authors confirm that all data underlying the findings are fully available without restriction.

\section{Authors' contributions}

MPC and JW conceived of this study and participated in the design of the study. MPC and CCS oversaw all aspects of the research. ZMG and JKW performed RNA extraction and real-time PCR analysis. LJS and XLC analyzed the microarray data and drew figures and tables. JW and ZMG performed statistic analysis and wrote the manuscript; ML revised it. All authors contributed to and have approved the final manuscript.

\section{Competing interests}

The authors declare that they have no competing interests.

\section{Ethics approval and consent to participate}

This study was approved by the Institutional Ethics Review Board of the First Affiliated Hospital of Wenzhou Medical University and the Ethics Committee of the Second Affiliated Hospital \& Yuying Children's Hospital of Wenzhou Medical University. Regarding the fetal cardiac tissues, written informed consent was obtained from the parents/guardians stating that the hearts could be used for research purposes and the fetal hearts were collected from Oct 1, 2014 to March 31, 2015 in the Second Affiliated Hospital \& Yuying Children's Hospital of Wenzhou Medical University. Regarding the adult cardiac tissues, written informed consent from the donor or the next of kin was obtained for the use of the sample in research and the adult hearts were collected from Oct 1 , 2014 to June 31, 2015 in the First Affiliated Hospital of Wenzhou Medical University. The hearts were studied in a completely anonymized fashion.

\section{Author details}

${ }^{1}$ Department of Cardiac Surgery, the First Affiliated Hospital of Wenzhou Medical University, Nanbaixiang, Shangcaicun, Wenzhou 325000, Zhejiang Province, People's Republic of China. ${ }^{2}$ Children's Heart Center, the Second Affiliated Hospital \& Yuying Children's Hospital, Institute of Cardiovascular Development and Translational Medicine, Wenzhou Medical University, No. 109, Xueyuan Road, Wenzhou 325000, Zhejiang Province, People's Republic of China. ${ }^{3}$ Tianjin Childrens' Hospital, Tianjin, People's Republic of China. ${ }^{4}$ Department of Transplantation, the First Affiliated Hospital of Wenzhou Medical University, Wenzhou, Zhejiang Province, People's Republic of China. ${ }^{5}$ Cardiac Regeneration Research Institute, Wenzhou Medical University, Wenzhou, Zhejiang Province, People's Republic of China. ${ }^{6}$ Department of Cardiology, the First Affiliated Hospital of Wenzhou Medical University, Wenzhou, Zhejiang Province, People's Republic of China.

Received: 19 August 2016 Accepted: 12 October 2016 Published online: 18 November 2016

\section{References}

1. Henderson DJ, Anderson RH. The development and structure of the ventricles in the human heart. Pediatr Cardiol. 2009;30:588-96.

2. Iruretagoyena Jl, Davis W, Bird C, Olsen J, Radue R, Teo Broman A, Kendziorski C, Splinter BonDurant S, Golos T, Bird I, Shah D. Metabolic gene profile in early human fetal heart development. Mol Hum Reprod. 2014;20(7):690-700.

3. Laflamme MA, Murry CE. Heart regeneration. Nature. 2011;473:326-35.

4. Mattick JS, Amaral PP, Dinger ME, Mercer TR, Mehler MF. RNA regulation of epigenetic processes. Bioessays. 2009;31:51-9.

5. Amaral PP, Clark MB, Gascoigne DK, Dinger ME, Mattick JS. IncRNAdb: a reference database for long noncoding RNAs. Nucleic Acids Res. 2011:39:D146-51.

6. Xu D, Yang F, Yuan J, Zhang L, Bi H, Zhou C, Liu F, Wang F, Sun S. Long noncoding RNAs associated with liver regeneration 1 accelerates hepatocyte proliferation during liver regeneration by activating Wnt/ $\beta$ Catenin signaling. Hepatology. 2013;58:739-51.

7. Zhang M, Gu H, Xu W, Zhou X. Down-regulation of IncRNA MALAT1 reduces cardiomyocyte apoptosis and improves left ventricular function in diabetic rats. Int J Cardiol. 2016;203:214-6.

8. Mattick JS. Long noncoding RNAs in cell and developmental biology. Semin Cell Dev Biol. 2011;22:327.

9. Cho KO, Hsieh J. The IncRNA Pnky in the brain. Cell Stem Cell. 2015:16:344-5.

10. Kornienko AE, Guenzl PM, Barlow DP, Pauler FM. Gene regulation by the act of long non-coding RNA transcription. BMC Biol. 2013:11:59.

11. Dinger ME, Pang KC, Mercer TR, Crowe ML, Grimmond SM, Mattick JS. NRED: a database of long noncoding RNA expression. Nucleic Acids Res. 2009;37:D122-6.

12. Klattenhoff CA, Scheuermann JC, Surface LE, Bradley RK, Fields PA Steinhauser ML, Ding H, Butty VL, Torrey L, Haas S. Braveheart, a long noncoding RNA required for cardiovascular lineage commitment. Cell. 2013:152:570-83.

13. Grote $P$, Wittler L, Hendrix D, Koch F, Währisch S, Beisaw A, Macura K, Bläss $\mathrm{G}$, Kellis M, Werber M. The tissue-specific IncRNA Fendrr is an essential regulator of heart and body wall development in the mouse. Dev Cell. 2013:24:206-14

14. Loewer S, Cabili MN, Guttman M, Loh YH, Thomas K, Park IH, Garber M, Curran M, Onder T, Agarwal S, et al. Large intergenic non-coding RNA-RoR modulates reprogramming of human induced pluripotent stem cells. Nat Genet. 2010;42:1113-7.

15. Pang KC, Frith MC, Mattick JS. Rapid evolution of noncoding RNAs: lack of conservation does not mean lack of function. Trends Genet. 2006:22:1-5.

16. Santoro F, Pauler FM. Silencing by the imprinted Airn macro IncRNA: transcription is the answer. Cell Cycle. 2013;12:711-2.

17. Soufan AT, van den Berg G, Ruijter JM, de Boer PA, van den Hoff MJ, Moorman AF. Regionalized sequence of myocardial cell growth and proliferation characterizes early chamber formation. Circ Res. 2006:99:545-52.

18. Ikenishi A, Okayama H, Iwamoto N, Yoshitome S, Tane S, Nakamura K, Obayashi T, Hayashi T, Takeuchi T. Cell cycle regulation in mouse heart during embryonic and postnatal stages. Dev Growth Differ. 2012;54:731-8.

19. Tane S, Kubota M, Okayama H, Ikenishi A, Yoshitome S, Iwamoto N, Satoh Y, Kusakabe A, Ogawa S, Kanai A, et al. Repression of cyclin D1 expression is necessary for the maintenance of cell cycle exit in adult mammalian cardiomyocytes. J Biol Chem. 2014;289:18033-44.

20. Paradis AN, Gay MS, Zhang L. Binucleation of cardiomyocytes: the transition from a proliferative to a terminally differentiated state. Drug Discov Today. 2014;19:602-9.

21. Lavine KJ, Yu K, White AC, Zhang X, Smith C, Partanen J, Ornitz DM Endocardial and epicardial derived FGF signals regulate myocardial proliferation and differentiation in vivo. Dev Cell. 2005;8:85-95.

22. Heallen T, Zhang M, Wang J, Bonilla-Claudio M, Klysik E, Johnson RL, Martin JF. Hippo pathway inhibits Wnt signaling to restrain cardiomyocyte proliferation and heart size. Science. 2011;332:458-61.

23. von Gise A, Lin Z, Schlegelmilch K, Honor LB, Pan GM, Buck JN, Ma Q Ishiwata T, Zhou B, Camargo FD, et al. YAP1, the nuclear target of Hippo signaling, stimulates heart growth through cardiomyocyte proliferation but not hypertrophy. Proc Natl Acad Sci U S A. 2012;109:2394-9.

24. Xin M, Kim Y, Sutherland LB, Qi X, McAnally J, Schwartz RJ, Richardson JA Bassel-Duby R, Olson EN. Regulation of insulin-like growth factor signaling by Yap governs cardiomyocyte proliferation and embryonic heart size. Sci Signal. 2011;4:ra70 
25. Gan J, Sonntag HJ, Tang MK, Cai D, Lee KK. Integrative analysis of the developing postnatal mouse heart transcriptome. PLoS One. 2015;10: e0133288.

26. Sun L, Xue H, Jiang C, Zhou H, Gu L, Liu Y, Xu C, Xu Q. LncRNA DQ786243 contributes to proliferation and metastasis of colorectal cancer both in vitro and in vivo. Biosci Rep. 2016;36(3):e00328.

27. Kino T, Hurt DE, Ichijo T, Nader N, Chrousos GP. Noncoding RNA Gas5 is a growth arrest and starvation-associated repressor of the glucocorticoid receptor. Sci Signal. 2010;3:ra8.

28. Jaeggi ET, Fouron J-C, Silverman ED, Ryan G, Smallhorn J, Hornberger LK. Transplacental fetal treatment improves the outcome of prenatally diagnosed complete atrioventricular block without structural heart disease. Circulation. 2004;110:1542-8.

29. Mourtada-Maarabouni M, Pickard M, Hedge V, Farzaneh F, Williams G. GAS5, a non-protein-coding RNA, controls apoptosis and is downregulated in breast cancer. Oncogene. 2009;28:195-208.

30. Liu Z, Wang W, Jiang J, Bao E, Xu D, Zeng Y, Tao L, Qiu J. Downregulation of GAS5 promotes bladder cancer cell proliferation, partly by regulating CDK6. PLoS One. 2013;8:e73991.

31. Leuranguer V, Monteil A, Bourinet E, Dayanithi G, Nargeot J. T-type calcium currents in rat cardiomyocytes during postnatal development: contribution to hormone secretion. Am J Phys Heart Circ Phys. 2000;279:H2540-8.

32. Cribbs LL, Lee J-H, Yang J, Satin J, Zhang Y, Daud A, Barclay J, Williamson $\mathrm{MP}$, Fox M, Rees M. Cloning and characterization of a1H from human heart, a member of the T-type Ca2+ channel gene family. Circ Res. 1998;83:103-9.

\section{Submit your next manuscript to BioMed Central and we will help you at every step:}

- We accept pre-submission inquiries

- Our selector tool helps you to find the most relevant journal

- We provide round the clock customer support

- Convenient online submission

- Thorough peer review

- Inclusion in PubMed and all major indexing services

- Maximum visibility for your research

Submit your manuscript at www.biomedcentral.com/submit

C) Biomed Central 